\title{
In situ-Diagnostik der Kontaktstelle ungehäuster Silizium- Leistungshalbleiter mittels Pyrometrie
}

\author{
Steffen Junker ${ }^{1}$, Volkmar Norkus ${ }^{1}$, Michael Schaulin ${ }^{2,}$ Gerald Gerlach ${ }^{1}$ \\ ${ }^{1}$ Institut für Festkörperelektronik, Technische Universität Dresden, Mommsenstr.15, 01069 Dresden, \\ Deutschland \\ 2Institut für Aufbau- und Verbindungstechnik der Elektronik, Technische Universität Dresden, \\ Mommsenstr.15, 01069 Dresden, Deutschland \\ steffen.junker@tu-dresden.de
}

\section{Zusammenfassung}

Diese Arbeit beschäftigt sich mit der Überprüfung der Kontaktstellen ungehäuster SiLeistungshalbleiter, die auf den IGBT-Modulen mittels einer Sinter- oder Lotverbindung aufgebracht werden. Vorgestellt wird in diesem Beitrag eine inlinefähige Messmethode, bei der ein gerichteter, zeitlich gesteuerter Heißluftstrom die Si-Oberfläche von oben erwärmt. Mit Hilfe eines Pyrometers erfolgt die zeitliche Erfassung der Temperatur an der Oberfläche der Bauelemente. Aus der Temperaturverteilung kann damit der Zustand der Kontaktstellen ermittelt werden.

Auf der Grundlage eines vereinfachten Modells zur direkten Anregung der Si-Oberfläche sowie auf der Basis von thermisch transienten Simulationen wurden die thermische Zeitkonstante und die absolute Temperaturdifferenz als Qualitätskriterien ermittelt. Hierfür wurde eine NiCr-HeizmäanderWiderstandsstruktur auf die Si-Oberfläche abgeschieden. Der Einfluss der Kontaktstelle kann anhand der beiden Parameter bewertet werden, da sich abhängig von der Homogenität der Kontaktstruktur unterschiedliche thermische Verläufe einstellen. Zur Validierung des Messplatzes wurden Messungen zur Reproduzierbarkeit der Ergebnisse durchgeführt. Der Beitrag liefert dabei einen möglichen Ansatz zur Bestimmung größerer Lufteinschlüsse im Kontaktbereich.

Keywords: Pyrometer,IGBT-Leistungsmodul,Kontaktstellendiagnostik,In situ-Diagnostik

\section{Einleitung und Motivation}

Die Sicherstellung der Funktionsfähigkeit von sogenannten Insulated Gate Bipolar Transistor-Modulen (IGBT) stellt eine große Herausforderung in der zerstörungsfreien Prüfung elektronischer Baugruppen dar. Wesentliche Bestandteile eines IGBT-Moduls sind ungehäuste Si-Chips, die mit Hilfe einer Sinter- oder Lotverbindung auf eine Direct Copper Bonded (DCB)-Keramik kontaktiert werden (Abb. 1) [1]. Die Si-Chips werden durch Al-Bonddrähte mit den umliegenden Leiterbahnen kontaktiert. Als Keramikwerkstoff wird meist Aluminiumnitrid (AIN) verwendet. Inhomogenitäten in der Kontaktstruktur, welche meist als Lufteinschlüsse (Voids) auftreten, haben einen besonders negativen Einfluss, da in deren Folge lokal hohe Temperaturen auftreten und daraus resultierend die Langzeitstabilität der gesamten Baugruppe reduziert wird [2] [3]. In Folge der Lufteinschlüsse erhöht sich der thermische
Widerstand $R_{\text {th }}$ der Baugruppe, was zu einer Überhitzung der Si-Chips führt und somit in der Zerstörung der gesamten Baugruppe resultieren kann.

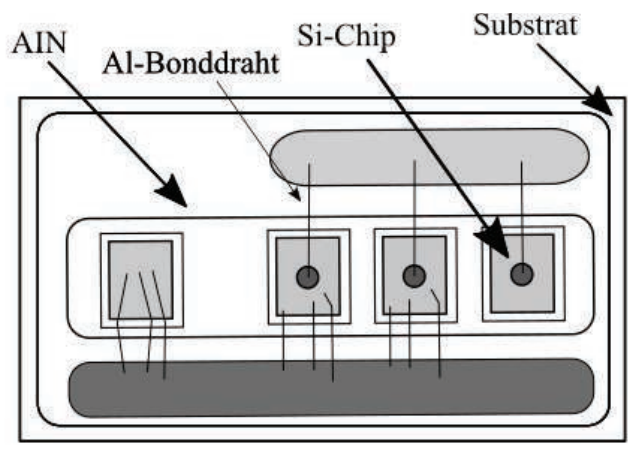

Abb. 1: Aufbau eines IGBT-Moduls.

Üblicherweise werden Kontaktstrukturen mit Röntgenstrahlmikroskopie, Ultraschallmikroskopie [4], aktiver-Thermografie [5] sowie durch 
Messung der thermischen Impedanz $Z_{\text {th }}$ auf Basis der Junction-Temperatur $T_{\mathrm{j}}$ [6] überprüft. Nachteile dieser Methoden sind jedoch die zum Teil hohen Prozess- und Wartungskosten und die fehlende inline-Tauglichkeit. Auf Grundlage der Aktiv-Thermografie wurde daher eine vereinfachte Messanordnung entwickelt, mit der sich Lufteinschlüsse in der Kontaktstruktur detektieren lassen.

\section{Messprinzip}

Das Messprinzip zur Bestimmung der Homogenität der Kontaktstruktur basiert auf der zeitlichen Aufnahme der Oberflächentemperatur To thermisch extern angeregter SiChips. Dabei werden die Si-Chips mit Hilfe einer geeigneten Anregungsenergie von oben erwärmt, d.h. thermisch angeregt. Ein Pyrometer misst die sich an der Oberfläche einstellende Erwärmung und die anschließende Abkühlung. Das verwendete Pyrometer besteht aus einem rauscharmen, hochauflösenden pyroelektrischen EinelementSensor, einer ebenso rauscharmen Auswerteelektronik und einer Chopper-Baugruppe.

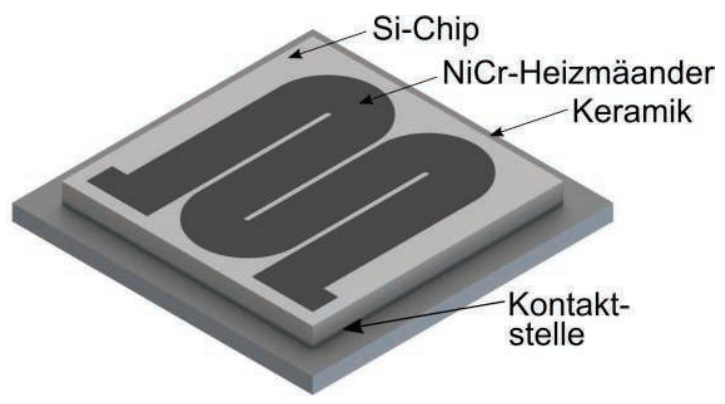

\section{Abb. 2: NiCr-Heizmäander Widerstandsstruktur.}

Für erste Versuche zur Beurteilung optimaler Charakterisierungsparameter erfolgte die thermische Anregung der Oberfläche durch eine aufgedampfte, elektrisch gespeiste $\mathrm{NiCr}$ Widerstandsheizstruktur (Abb. 2). Diese Heizstruktur ermöglicht es, gezielte Wärmeimpulse in das Materialsystem zu leiten.

Eine kostengünstige, inlinefähige Lösung ergibt sich bei Anregung der Oberfläche mit Hilfe eines Heißluftgebläses (Abb. 3a, 3b). Die IGBT-Module befinden sich auf einem thermostatisierbaren Probenträger, um die Ausgangstemperatur zu erhöhen. Dadurch erhöht sich der Signal-Rausch-Abstand des Pyrometers. Ein gerichteter Heißluftstrom, erzeugt von einem Heißluftgebläse, erwärmt die Si-Chips von oben. Das Pyrometer misst die Temperaturänderung an der Oberfläche des Si-Chips und die sich anschließende,

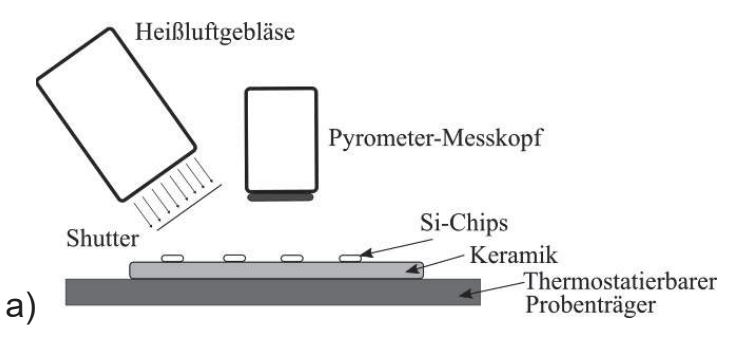

Abb. 3: Messaufbau a) Prinzipskizze, b) Foto.

durch Ausgleichvorgänge im Materialsystem einstellende Abkühlung. Wichtig bei der Wahl einer geeigneten Anregungsenergie ist, dass diese modulierbar sein sollte. In Folge der hohen spezifischen Wärmeleitfähigkeit $\lambda$ von Silizium $(150 \mathrm{~W} /(\mathrm{m} \bullet \mathrm{K}))$ resultieren entsprechend sehr kurze thermische Anregungszeiten [7].

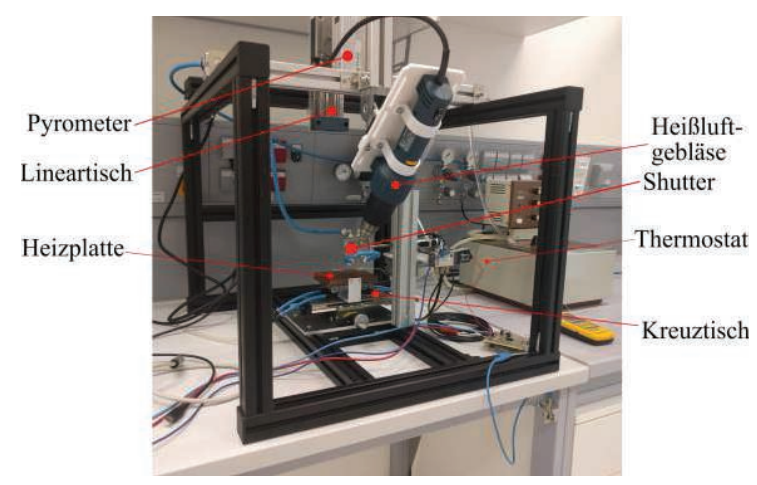

Abb. $3 b$

Wird das zu untersuchende System nicht zeitlich gepulst angeregt, ist die thermische Sprungantwort zusätzlich von anderen Materialparametern des Systems abhängig. Ein Maß zur Bestimmung der Anregungszeit ist die thermische Diffusionslänge $\mu$ :

$\mu=\sqrt{\frac{\alpha}{\pi * f}}$,

mit der Temperaturleitfähigkeit $\alpha$ und der Frequenz $f$ [8]. Für eine Frequenz $f=100 \mathrm{~Hz}$ beträgt die thermische Diffusionslänge $\mu=$ $0,54 \mathrm{~mm}$. Idealerweise beträgt die Anregungszeit zur Überprüfung der Kontaktstellen in IGBT-Modulen für geometrische Si-Dicken von $550 \mu \mathrm{m}$ ca. $60 \mathrm{~ms}$.

In der in Abb. 3 dargestellten Messanordnung lässt sich die Dauer des zeitlichen Wärmeimpulses durch ein mechanisch gesteuertes und zudem gekühltes ShutterSystem variabel einstellen. $\mathrm{Zu}$ beachten ist jedoch die Trägheit des mechanischen Systems im Vergleich zur Anregung der SiChips mit der Widerstandsheizstruktur von Abb. 2, wodurch die minimal realisierbare 
Anregungszeit begrenzt wird. Aufgrund der Mikrofonie-Abhängigkeit pyroelektrischer Infrarotsensoren ist es notwendig, dass mechanisch gesteuerte Shutter-System vom Messplatz zu entkoppeln, um auftretende Querempfindlichkeiten zu vermeiden [9].

Gewöhnlich werden in der Aktiv-Thermografie Kontaktstrukturen mit Hilfe einer hochauflösenden, gekühlten Thermografiekamera bei einer Bildfrequenz $f_{m}$ von bis zu $100 \mathrm{~Hz}$ und einer Auflösung von 640 x 512 Pixel untersucht [5] [10]. Die Darstellung der thermischen Sprungantwort angeregter SiChips, aufgenommen mit einer gekühlten Infrarotkamera (FLIR Cedip Silver 660), ist in Abb. 4 dargestellt. Zu erkennen ist die unterschiedliche Wärmeverteilung an der SiOberfläche in Abhängigkeit der Homogenittät der Kontaktstelle.
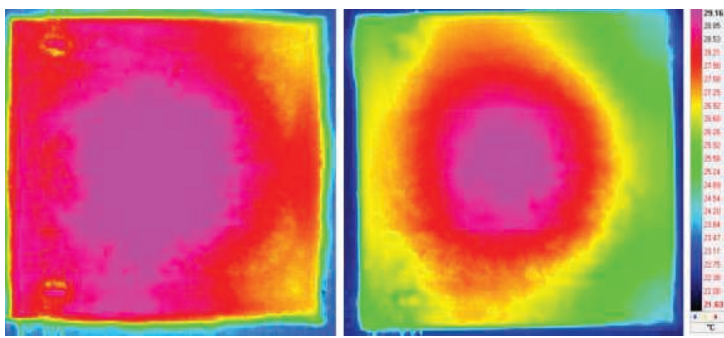

Abb. 4 Thermisches Verhalten von Si-Chips mit $0 \%$ (links) und 96\% (rechts) Lufteinschlüssen nach einer thermischen Anregung.

Durch die Verwendung eines Pyrometers reduzieren sich die Prozess- und Systemkosten aber auch die geometrische Messauflösung auf 1 Pixel. Aus diesem Grund ist eine exakte Lokalisierung der Defekte im Material nicht möglich. Abhängig von der Homogenität des Kontaktmaterials variieren jedoch die zeitlich abhängigen Ausgleichsvorgänge im Kontaktmaterial, welche weiterhin mit dem verwendeten Pyrometer detektierbar bleiben. Ursache dieses Effekts ist die Isolationseigenschaft von Luft, welche auf die geringe Wärmeleitfähigkeit $\lambda_{\text {Luft, }}$ die bei Raumtemperatur $26,2 \mathrm{~mW} /(\mathrm{m} \cdot \mathrm{K})$ beträgt, zurückzuführen ist [11]. Mit zunehmendem Anteil von Luft im Kontaktmaterial dominieren die Einschlüsse deutlich die Ausgleichsvorgänge. Die Auswertung der Messergebnisse kann hinsichtlich der thermischen Zeitkonstante:

$$
\tau=T_{\min }+\left[\left(T_{\max }-T_{\min }\right) * 0,368\right]
$$

und der absoluten Temperaturdifferenz:

$$
\Delta T=T_{\max }-T_{\min }
$$

erfolgen. Dabei nimmt mit zunehmendem Luftanteil im Materialsystem sowohl die Temperaturdifferenz $\Delta T$ als auch die thermische Zeitkonstante $T$ zu. Eine Auswertung hinsichtlich der absoluten Temperaturdifferenz $\Delta T$ kann nur bei konstanter Anregungszeit $t$ impuls erfolgen.

\section{Aufbau der Messproben}

Zur Verifizierung des entwickelten Messprinzips wurden Messproben mit einer definierten Größe des Lufteinschlusses in der Kontaktstruktur hergestellt. Aus diesem Grund wurden Messproben angefertigt, die definierte Lufteinschlüsse in der Kontaktstruktur aufweisen (Abb. 5, 6). Zur besseren Reproduzierbarkeit der Testproben wurden die SiChips (Dicke $550 \mu \mathrm{m}$ ) mittels eines AgLeitklebers (Dicke $100 \mu \mathrm{m}$ ) auf einer Keramik (Dicke $670 \mu \mathrm{m}$ ) kontaktiert. Der Leitkleber besitzt ähnliche elektrische und thermische Eigenschaften wie standardmäßig verwendete Lote $\left(\lambda_{\text {Kleber }}=30 \mathrm{~W} /(\mathrm{m} \cdot \mathrm{K})\right)$.

Silizium $(550 \mu \mathrm{m}) \quad$ Ag-Leitkleber $(100 \mu \mathrm{m}) \quad$ Keramik $(670 \mu \mathrm{m})$ Absorptions-

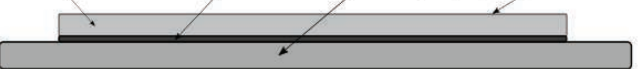

\section{Abb. 5: Struktureller Schichtaufbau der Messproben.}

Dabei wurde der Ag-Leitkleber unterschiedlich strukturiert auf die Keramikoberfläche aufgebracht (Abb. 6).

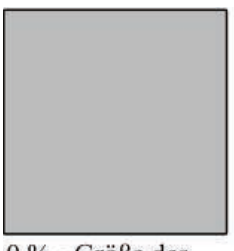

$0 \%$ - Größe des Lufteinschlusses

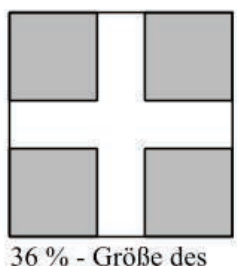

$36 \%$ - Größe des Lufteinschlusses

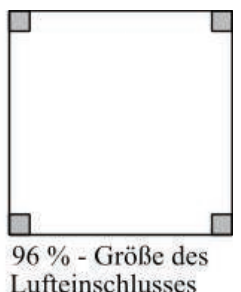

Lufteinschlusses
Abb. 6 Untersuchte Kontaktstrukturen mit unterschiedlichen Luftanteilen in der Kontaktstelle.

Da Lage und Größe der Lufteinschlüsse bekannt waren, können konkrete obere Grenzwerte für die Zeitkonstante $T$ und der Temperaturdifferenz $\Delta T$ festgelegt werden.

Dadurch, dass Silizium in einem Wellenlängenbereich von $8 \mu \mathrm{m}$ bis $14 \mu \mathrm{m}$ spektral transparent ist, wurden alle Proben auf der Oberfläche mit einer Absorptionsschwarzschicht beschichtet. Hiermit tritt im genannten Wellenlängenbereich ein Emissionsgrad von ca. 0,96 auf, der somit die Bestimmung der Temperatur an der SiOberfläche ermöglicht. 


\section{Thermisch transiente Simulation des Silizium-Chips}

Zur Überprüfung und Veranschaulichung der thermischen Ausgleichsprozesse im Materialsystem wurden dreidimensionale Simulationen durchgeführt. Die thermisch transiente Analyse erfolgte mit der Finite-Elemente-Software ANSYS. Das verwendete geometrische Modell ist in Abb. 7a dargestellt.

a)

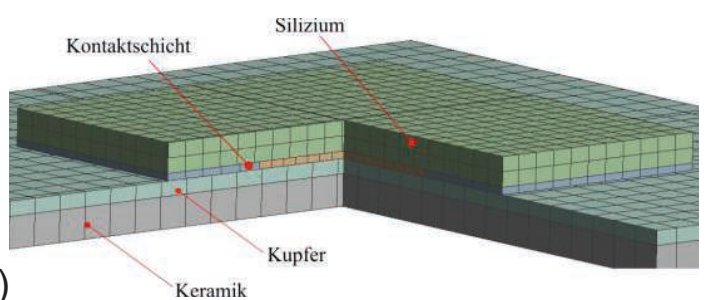

Abb. 7: Dreidimensionale Darstellung des Simulationsmodells (a) Schichtaufbau, (b) ohne und (c) mit 25\% Luftanteil in der Kontaktschicht.

In den nachfolgenden Simulationsdarstellungen (Abb. 7b, 7c) ist die Wärmeverteilung eines Teilbereiches des IGBT-Moduls dargestellt, wenn dieses impulsartig $\left(\Delta T=6 \mathrm{~K}, t_{\text {Impuls }}=60 \mathrm{~ms}\right)$ thermisch angeregt wird. $\mathrm{Zu}$ erkennen sind zwei unterschiedliche Ausbreitungsvorgänge infolge der unterschiedlichen Luftanteile in der Kontaktschicht des Systems.

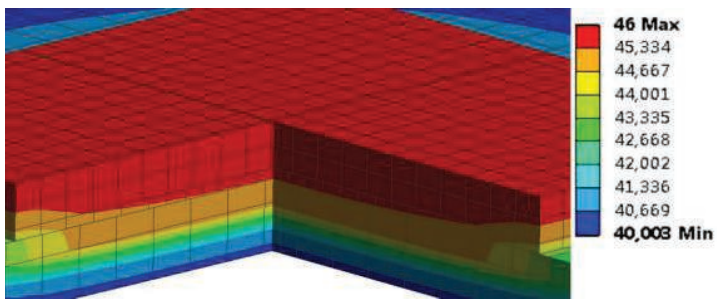

Abb. $7 b$

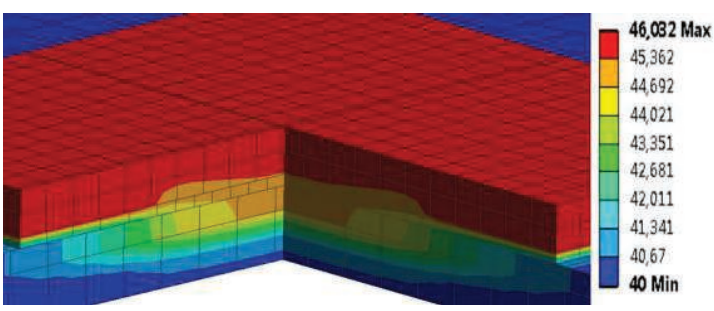

Abb. $7 c$

Die Ergebnisse zeigen eine deutliche inhomogenere Wärmeausbreitung in der Kontaktschicht, wenn diese Lufteinschlüsse beinhaltet. Ursache ist der große Wärmeleitfähigkeitsunterschied $\lambda$ beider Materialien $\left(\lambda_{\text {Lot }}=48 \mathrm{~W} /(\mathrm{m} \bullet \mathrm{K})\right)$. Abhängig vom Luftanteil der Kontaktschicht variieren die maximalen Temperaturen an der Si-Oberfläche und somit auch die Dauer des thermischen Ausgleichsprozesses. Dies zeigt, dass Inhomogenitäten in der Kontaktstelle zu einer thermischen Überlastung der gesamten Baugruppe führen können.

\section{Messergebnisse}

\section{NiCr-Heizmäander}

Die Messungen erfolgten mit den drei unterschiedlichen Messproben aus Abb. 6. Ziel der Messungen war die Validierung optimaler Prozess- und Auswerteparameter, die für die Bestimmung des Luftanteils in der Kontaktstruktur notwendig sind. Die Widerstandsheizstruktur wurde mit einem Spannungsimpuls von $42 \mathrm{~V}$ für $70 \mathrm{~ms}$ elektrisch angeregt. Die Temperaturverläufe sind in Abb. 8 und eine Übersicht der Auswerteparameter in Tab. 1 dargestellt. Zur Verständlichkeit ist in Abb. 8 die absolute Temperaturdifferenz $\Delta T$ und die thermische Zeitkonstante $T$ für die Messprobe mit einem 96\% Lufteinschluss grafisch gekennzeichnet. $T_{\max }$ ergibt sich aus der maximalen Erwärmung der Probe, während $T_{\min }$ sich aus der mittleren Durchschnittstemperatur des eingeschwungenen Zustands zusammensetzt. Aus Abb. 8 lässt sich erkennen, dass mit größerem Luftanteil in der Kontaktstelle die absolute Temperaturdifferenz $\Delta T$ und die thermische Zeitkonstante $T$ ansteigen.

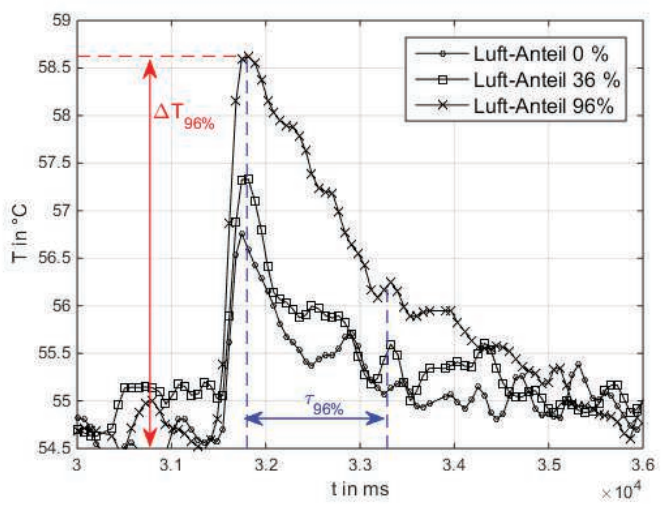

Abb. 8: Temperaturverlauf der Si-ChipOberfläche bei Erwärmung mit NiCrHeizmäander für $70 \mathrm{~ms}$

Beide Parameter eignen sich damit als Indikatoren zur Bestimmung größerer Lufteinschlüsse in der Kontaktstelle. Am deutlichsten sind diese Ergebnisse mit zunehmendem Luftanteil.

Zur schnelleren und eindeutigeren Beurteilung der Homogenität einer Kontaktstelle eignet sich ein maximaler Grenzwert. Eine Überschreitung dieses Grenzwertes deutet auf 
Inhomogenitäten der Kontaktstelle. Basierend auf den ermittelten Ergebnissen für eine nahezu vollflächige homogene Kontaktstelle, kann ein solcher Grenzwert festgelegt werden.

Tab. 1: Thermische Zeitkonstante $t$ und absolute Temperaturdifferenz $\Delta T$ der Testproben, angeregt mittels der Widerstandsheizstruktur von Abb. 2

\begin{tabular}{|c|c|c|}
\hline $\begin{array}{c}\text { Luft- } \\
\text { anteil } \\
{[\%]}\end{array}$ & $\begin{array}{c}\text { Thermische } \\
\begin{array}{c}\text { Zeitkonstante } T \\
{[\mathrm{~ms}]}\end{array}\end{array}$ & $\begin{array}{c}\text { Absolute } \\
\text { Temperatur- } \\
\text { differenz } \Delta T \\
{[\mathrm{~K}]}\end{array}$ \\
\hline 0 & 909 & 2,04 \\
\hline 36 & 1128 & 2,42 \\
\hline 96 & 1488 & 3,81 \\
\hline
\end{tabular}

\section{Heißluftgebläse}

Auf der Grundlage des oben beschriebenen Modells erfolgte die Anregung der SiOberfläche mit Hilfe eines Heißluftgebläses. Die Untersuchungen wurden unter identischen Umgebungsbedingungen wie zuvor durchgeführt, allerdings mit einer deutlich längeren Anregungszeit des Materialsystems. Diese betrug durch das verwendete Shutter-System $200 \mathrm{~ms}$. Allerdings ist die effektive Anregungszeit deutlich kleiner, da der Wärmestrom vom Heißluftgebläse die Strecke zur Messoberfläche zurücklegen muss.

In Abb. 9 und Tab. 2 sind die Ergebnisse der Versuche dargestellt. Wie auch bei der Impulsanregung durch eine Widerstandsstruktur ergeben sich ähnliche Verläufe des zeitlichen Temperaturverlaufs.

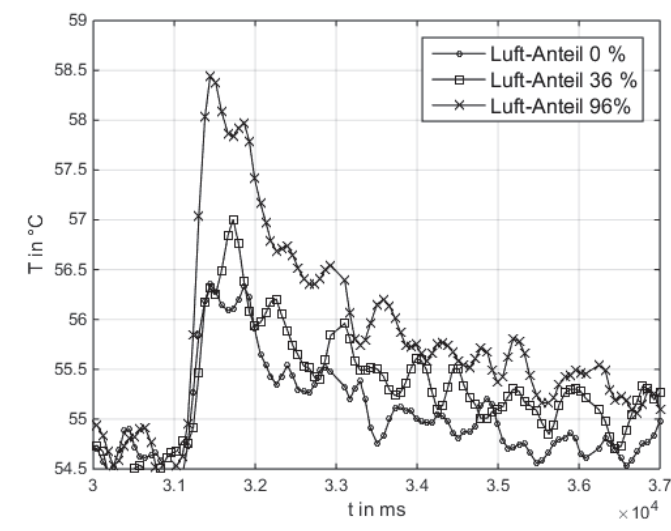

Abb. 9: Temperaturverlauf der Si-ChipOberfläche bei Erwärmung mit Heißluftgebläse für 200 ms.

Auch hier führen größere Inhomogenitäten in der Kontaktstelle zu einem Anstieg der absoluten Temperaturdifferenz $\Delta T$ und der thermischen Zeitkonstante $T$.
Tab. 2: $\quad$ Thermische Zeitkonstante $t$ und absolute Temperaturdifferenz $\Delta T$ der Testproben, angeregt mittels Heißluftgebläse

\begin{tabular}{|c|c|c|}
\hline $\begin{array}{c}\text { Luft- } \\
\text { anteil } \\
{[\%]}\end{array}$ & $\begin{array}{c}\text { Thermische } \\
\text { Zeitkonstante } \\
T[\mathrm{~ms}]\end{array}$ & $\begin{array}{c}\text { Absolute } \\
\text { Temperaturdifferenz } \\
\Delta T[\mathrm{~K}]\end{array}$ \\
\hline 0 & 1288 & 2,20 \\
\hline 36 & 1688 & 2,71 \\
\hline 96 & 2224 & 3,84 \\
\hline
\end{tabular}

\section{Reproduzierbarkeit}

Zur Validierung des Messverfahrens wurden insgesamt 72 Messungen, verteilt auf drei Testproben, zur Reproduzierbarkeit der Ergebnisse durchgeführt. In der Abb. 10 sind beispielhaft vier Messergebnisse für die Messprobe mit einer $96 \%$ Größe des Lufteinschlusses in der Kontaktschicht dargestellt. Die maximalen Temperaturen sind für jede der vier Messungen nahezu identisch. Es kommt jedoch zu Abweichungen im Abkühlverhalten, die auf das Sensorrauschen zurückzuführen sind. Eine Zusammenfassung der 72 Messungen zur Reproduzierbarkeit ist in der Tab. 3 und 4 dargestellt.

Tab. 3: Mittelwerte der thermischen

Zeitkonstanten und der absoluten

Temperaturdifferenzen des mittels eines Heißluftgebläses erwärmten Testproben

\begin{tabular}{|c|c|c|}
\hline $\begin{array}{c}\text { Luft- } \\
\text { anteil } \\
{[\%]}\end{array}$ & $\begin{array}{c}\text { Mittelwert der } \\
\text { thermischen } \\
\text { Zeitkonstante } \\
T[\mathrm{~ms}]\end{array}$ & $\begin{array}{c}\text { Mittelwert der } \\
\text { absoluten } \\
\text { Temperatur- } \\
\text { differenz } \Delta T \\
{[\mathrm{~K}]}\end{array}$ \\
\hline 0 & 1301 & 2,28 \\
\hline 36 & 1709 & 2,60 \\
\hline 96 & 2184 & 3,55 \\
\hline
\end{tabular}

Ein Hauptproblem der vorgestellten Messmethode ist die Erfassung kleinerer Lufteinschlüsse in der Kontaktschicht. Wie in den Simulationsergebnissen gezeigt wurde, resultieren aus größeren Inhomogenitäten größere maximale Temperaturänderungen an der Silizium-Oberfläche. Aus diesem Grund ergeben sich für kleinere Lufteinschlüsse größere Messunsicherheiten, da das resultierende Signal im pyroelektrischen Sensor kleiner wird. Die Standardabweichung für die ermittelte thermische Zeitkonstante ist in diesem Bereich deutlich größer als bei höheren Temperaturen (Tab. 4). Des Weiteren kann mit dieser Messmethode der Wärmefluss $\Phi$, der in das Materialsystem eingebracht wird, 
nicht exakt bestimmt werden, da die reale thermische Anregungszeit unbekannt ist.

Tab. 4: Standardabweichung der thermischen Zeitkonstanten und der absoluten Temperaturdifferenzen des mittels eines Heißluftgebläses erwärmten Testproben

\begin{tabular}{|c|c|c|}
\hline $\begin{array}{c}\text { Luft- } \\
\text { anteil } \\
{[\%]}\end{array}$ & $\begin{array}{c}\text { Standard- } \\
\text { abweichung } \\
\text { der } \\
\text { thermischen } \\
\text { Zeitkonstante } \\
\text { [ms] }\end{array}$ & $\begin{array}{c}\text { Standard- } \\
\text { abweichung } \\
\text { der absoluten } \\
\text { Temperatur- } \\
\text { differenz } \Delta T \\
{[\mathrm{~K}]}\end{array}$ \\
\hline 0 & 334 & 0,23 \\
\hline 36 & 208 & 0,34 \\
\hline 96 & 231 & 0,30 \\
\hline
\end{tabular}

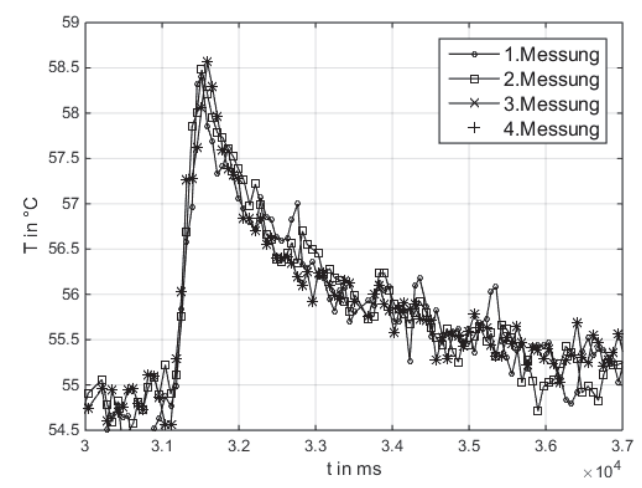

Abb. 10: Temperaturverlauf der Si-ChipOberfläche für einen Luftanteil von $96 \%$ in der Kontaktstelle.

Infolgedessen ist eine Überschreitung der maximalen Anregungszeit möglich und muss beachtet werden. Inhomogene Kontaktstellen lassen sich aber identifizieren, wenn ein maximaler Grenzwert überschritten wird.

\section{Zusammenfassung und Ausblick}

In diesem Beitrag wurde eine inlinefähige Methode zur Untersuchung der Kontaktstelle von IGBT-Leistungshalbleitern mit dem Substrat vorgestellt. Anhand eines einfachen Wärmeleitungs-Modells und thermisch transienten Simulationen konnte Zusammenhang zwischen Inhomogenitäten an der Kontaktstelle und den Temperaturdifferenzen an der Silizium-Oberfläche gezeigt werden. Zukünftige Arbeiten sollen ein elektrisch gesteuertes Shutter-System verwenden, da sich so kürzere Anregungszeiten und somit geringere Abweichungen realisieren lassen. Anstelle des Heißluftgebläses könnte der
Wärmeimpuls durch eine Hochleistungs-LED oder mit Hilfe eines Lasers erzeugt werden.

\section{Literaturnachweis}

[1] N.Y.A. Shammas, Present problems of power module packaging technology, Microelectronics Reliability 43, 519-527 (2003); doi: 10.1016/S0026-2714(03)00019-2

[2] N.Y.A. Shammas, et al., Finite element modelling of thermal fatigue effects in IGBT modules, IEE Proceedings-Circuits, Devices and Systems 148, 95-100 (2001); doi: 10.1049/ip-cds:20010293

[3] R.O. Carlson, et al., Voids, cracks, and hot spots in die attach, Reliability Physics Symposium 21, 138-141 (1983); doi: 10.1109/IRPS.1983.361974

[4] S. Brand, et. al., Non-Destructive Assessment of Reliability and Quality related Properties of Power Electronic Devices for the In-Line Application of Scanning Acoustic Microscopy, International Conference on Integrated Power Electronics Systems 9, 1-6 (2016)

[5] C. Maierhofer, et al., Non-destructive testing of Cu solder connections using active thermography, NDT \& E International 52, 103111 (2012); doi: 10.1016/j.ndteint.2012.07.010

[6] B. Ji, et al., In situ diagnostics and prognostics of solder fatigue in IGBT modules for electric vehicle drives, IEEE Transactions on Power Electronics 30, 1535-1543 (2014); doi: 10.1109/TPEL.2014.2318991

[7] H.R. Shanks, et al., Thermal conductivity of silicon from 300 to $1400 \mathrm{~K}$, Physical Review 130.5, 1743-1748 (1963); doi: 10.1103/PhysRev.130.1743

[8] R. Arndt, et al., Zerstörungsfreie Ortung von Fehlstellen und Inhomogenitäten in Bauteilen mit der Impuls-Thermografie, Bautechnik 81, 786-793 (2004); doi: 10.1002/bate.200490185

[9] G. Gerlach, et al., Packaging influence on acceleration sensitivity of pyroelectric infrared detectors, Proceeding of the 6th IEEE CPMT Conference on IEEE, 297-302 (2004); doi: 10.1109/HPD.2004.1346715

[10] M. Schaulin, et al., Beiträge zur In-Situ Sensorik für die Inspektion von Kontaktstellen an Leistungshalbleitern, MikroSystemTechnik Kongress, 480-482 (2017)

[11] E.W. Lemmon, et al., Viscosity and thermal conductivity equations for nitrogen, oxygen, argon, and air, International Journal of Thermophysics 25.1, 21-69 (2004); doi: 10.1023/B:IJOT.0000022327.04529.f3 